\title{
EXPLOITATION PASTORALE ET COMPTE D'EXPLOITATION
}

PAR

\author{
J. de LARRÉ de La DORIE
}

Ingénieur Principal des Eaux et Forêts

La récolte de l'herbe par le pâturage constitue, en haute montagne. du fait de la pente et de l'altitude, le seul mode d'exploitation possible de terrains portant sur des zones étendues.

Si cette activité n'est pas celle qui, même dans ces régions, met en jeu les plus grandes sommes de valeurs consommées et produites localement, elle en demeure souvent la plus visible et la plus typique: elle retient, dès le premier abord, l'attention de tout observateur de la montagne.

Par ailleurs c'est, à tous égards, une spéculation marginale:

- au point de vue géographique, les exploitations de pâturages en haute montagne occupent les confins de l'aire retenue par la culture ou réservée à la forêt;

- au point de vue sociologique, elles intéressent des populations dont l'habitat est situé aux extrémités supérieures des vallées;

- au point de vue économique proprement dit, elles ont souvent des difficultés pour maintenir leurs prix de revient dans les limites de leurs prix de vente.

Il y a là une activité qui apparaît actuellement précaire quoiqu'elle ait eu une existence multi-séculaire.

On comprend que, dans ces conditions, depuis que l'homme a inventé la science économique, l'évolution passée, présente et future 
des exploitations pastorales en haute montagne, du fait de leur étendue, de leur variété et de leur état actuel, soit en principe un sujet de choix pour l'économiste.

Et en effet, au nom de l'Economie, nombre d'auteurs ont traité cette question: il n'est pas de forestier qui n'ait eu connaissance des problèmes de l'économie pastorale en haute montagne, en fonction d'études classiques sur l'équilibre sylvo-pastoral.

Si cependant on examine, tant les rapports sur les situations particulières d'exploitations déterminées, que les exposés faits en forme de thèses générales, on y trouve des observations intéressantes sur les données techniques de l'élevage et sur la condition sociale des exploitants, mais on regrette de n'y relever que très peu de données économiques proprement dites, c'est-à-dire concernant la consommation de biens et de services en vue de produire d'autres biens et d'autres services.

Il est vrai que les auteurs traitant de la haute montagne, n'ont retenu plus ou moins consciemment que les effets du relief sur l'exploitation pastorale et non l'exploitation pastorale elle-même. I1 résulte de ce fait que leurs conclusions manquent souvent de portée pratique.

Or, du point de vue économique, une exploitation pastorale est: - d'abord, une " exploitation );

- ensuite, une “ exploitation d'élevage »;

- et enfin seulement, une " exploitation d'élevage en haute montagne $)$.

C'est en partant de ce point de vue que je me permets de suggérer un plan pour l'étude véritablement économique d'une exploitation pastorale donnée.

Je dirai tout cle suite que la méthode préconisée n’a rien d'original: beaucoup y ont sans cloute déjà pensé, mais il est cle fait que dans de très nombreux cas particuliers où son emploi se serait imposé, de même que dans cles thèses d’intérêt général sur ce sujet, je ne l'ai jamais constaté.

Sans cloute, dans ces conditions, est-il utile de la préciser: elle consiste à établir un compte d'exploitation probalsle (évaluation des charges et des produits) pour l'année au cours de laquelle l'étucle est faite et ceci en s’inspirant cles charges et produits de l'année précédente pour les exploitations en cours de fonctionnement.

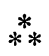

Les pâturages de haute montagne ne donnant de production que par l'intermédiaire d'un troupeau et celui-ci ne vivant d'ailleurs sur le pâturage que pendant une partie seulement du cycle de produc- 
tion annuel, c'est le « compte d'exploitation du troupeau ) que l'on cherchera à établir en évaluant:

a) Les charges probables de l'exploitation de l'année:

- Valeur du troupeau au $1^{\mathrm{er}} / 1 / 19$.. ...........

- Valeur des fourrages et des aliments en stock au $1^{\text {er }} / 1 / 19 . . \ldots \ldots \ldots \ldots \ldots \ldots \ldots \ldots$

- Achats de bétail au cours de l'année 19.. ....

Charges de l'exploitation dans la vallée:

- Achats d'aliments et de fourrages ..........

- Frais d'entretien et de traite du bétail

- Travaux d'entretien de l'étable de la vallée ....

- Amortissement de l'étable de la vallée

Charges de l'exploitation au pâturage:

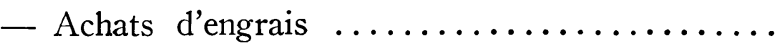

- Frais d'entretien et de traite du bétail .......

- Frais de transport de la vallée au pâturage et

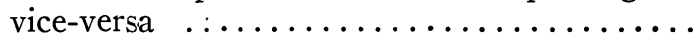

- Travaux d'entretien du chalet, des clôtures, du chemin d'accès et de la pelouse ............

- Amortissement du chemin d'exploitation (en 5 à 30 ans)

- Amortissement du chalet (en 10 à 20 ans) ....

- Amortissement des clôtures et d'autres installations (en 5 à 15 ans) $\ldots \ldots \ldots \ldots \ldots \ldots$.

Total des charges.

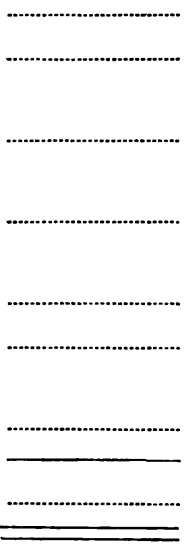

N.B. - Chacune de ces rubriques peut donner lieu à un commentaire, tant sur la nature des valeurs à y inscrire, que sur leur mode d'évaluation.

Je noterai seulement ici que:

- les travaux d'entretien sont ceux qui compensent l'usure due à l'usage de l'installation (pour un chemin, par exemple: comblement des ornières, curage des fossés);

- les amortissements correspondent à l'importance de la dépréciation non compensée par l'entretien, résultant du vieillissement dépendant de la nature de l'équipement, indépendamment de tout usage (pour un chemin, par exemple: le coût de la réparation de la désagrégation de la chaussée due à la nature du sol ou au climat local). 
b) Les produits probables de l'exploitation de l'année:

- Valeur du troupeau au 31/12/19.. .........

- Valeur des fourrages et aliments en stock au $31 / 12 / 19$..

- Ventes de lait et de produits dérivés

- Ventes de viande sur pied

- Ventes de laine

- Ventes de fourrage ...

Total des produits

c) Les résultats (pertes ou profits) probables de l'exploitation de l’année:

Par différence, on déterminera:

Produits moins Charges = Profit probable de l'exploitation de l'année, ou

Charges moins Produits $=$ Perte probable cle l'exploitation de l'année.

S'il y a profit, on examinera, s'il peut couvrir :

- l'annuité de remboursement d'emprunt d'amélioration éventuellement déjà souscrit,

- la valeur du loyer du pâturage et des bâtiments d'exploitation,

- le profit normal des propriétaires du troupeau.

Dès lors, l'emploi du compte d'exploitation probable se conçoit aisément.

En présence d'un projet de modification du mode d'exploitation (modification de la composition du troupeau, de l'étendue à pâturer, etc...) ou d'un projet de transformation de l'équipement (au moyen d'une aide financière extérieure), on déterminera la nature et l'évaluation des "Charges ) et des " Produits ) devant disparaître ou apparaître du fait de la transformation projetée du mode d'exploitation.

Ces " Charges » et (" Produits ) seront cumulés avec les " Charges » et les " Produits » analysés et on déterminera ainsi de nouveaux ("Résultats ) probables - (Profits ) ou " Pertes ) que l'on comparera avec les " Résultats » analysés par l'établissement du compte. 
Ainsi, le raisonnement conduisant aux directives d'action sera-t-il constamment soutenu et conservé par la rédaction du dit compte.

Les difficultés d'évaluations permettront notamment d'apprécier la rigueur et l'objectivité de ce raisonnement.

Pourquoi cette méthode d'analyse n'est-elle pas couramment appliquée? Et pourquoi serait-il difficile de la prendre en considération?

J'y vois pour ma part deux motifs:

$1^{\circ}$ Les différentes rubriques, en apparence anodines, des catégories de valeurs mises en jeu (notamment en ce qui concerne les charges) recouvrent en réalité des notions assez abstraites. Elles supposent que l'on ait une vue claire par exemple:

a) des valeurs qui sont comprises ou exclues de la spéculation économique envisagée, ce qui peut être complexe du fait que l'exploitation pastorale peut être poursuivie en commun par plusieurs propriétaires de bétail, ou au contraire ne constitue qu'une partie de l'activité d'un éleveur;

b) des différences fondamentales entre un compte d'exploitation et un compte de recettes et dépenses (Caisse ou Banque);

c) de la différence de nature essentielle entre les dépenses et recettes d'Equipement et les dépenses et recettes d'Exploitation;

d) de la nature et du mode d'évaluation des amortissements; etc...

Cependant, il ne manque pas de jeunes éleveurs qui peuvent assimiler facilement ces notions. En outre, ceux-ci disposent de conseils éclairés et bénévoles.

$2^{\circ}$ Ayant l'impression, à juste titre, qu'un tel compte donne une description très réaliste de l'exploitation, il est compréhensible que l'exploitant ne tienne pas à exposer son cas à un public plus ou moins étendu sauf si, exceptionnellement, il est satisfait de diriger une exploitation vedette, pilote ou témoin.

En fait, si le compte d'exploitation n'est destiné qu'au seul usage personnel de l'exploitant, il n'y a pas lieu de soulever cette objection.

Si le compte d'exploitation est destiné à un tiers dont, par exemple, l'exploitant sollicite le concours, ce compte d'exploitation n'est connu que par l'exploitant emprunteur et son prêteur, celui-ci ne devant s'en servir que dans leurs rapports réciproques. 
Si le compte d'exploitation est destiné à faire l'objet d'une étude d'intérêt général, rien ne s'oppose à se servir de celui-ci d'une façon anonyme.

Mais, outre ces deux motifs, jouant contre l'établissement du compte d'exploitation, il en est un autre plus général en la matière:

- Pourquoi, dira-t-on, se préoccuper de rentabilité en matière d'exploitation pastorale: celle-ci doit être maintenue en tout état de cause en vue d'éviter la transformation de la haute montagne en désert, sympathique point de vue de ceux qui sont attachés malgré tout à une activité à l'échelle de l'initiative individuelle se développant dans un milieu parfois physiquement exaltant.

Il faut observer à ce sujet que les résultats d'une activité économique, que l'on en ait conscience ou non, existent en tout état de cause. Mieux vaut, dans ces conditions, analyser celle-ci et déterminer ses résultats surtout s'il s'agit d'un déficit: c'est alors l'opération fondamentale pour rechercher et appliquer, s'il est possible, le moyen de transformer le déficit en bénéfice et d'assurer la poursuite de l'exploitation. Au surplus, pour combler raisonnablement un déficit délibérément accepté, au moyen d'un apport extérieur à l'exploitation, il faut connaître le montant de ce déficit.

Je ne peux clore ces quelques notes sans préciser que, m'inspirant de l'adage " les conseilleurs ne sont pas les payeurs », je n'ai pas entendu ci-dessus apprendre aux exploitants pastoraux à connaitre leur exploitation: ils la connaissent mieux que quiconque.

Mon propos est seulement de leur suggérer un mode d'expression correct de leur situation, afin de leur permettre de mieux la préciser et de justifier leurs prétentions éventuelles.

Erratum Revue Forestière Française, octobre 1964

Page 743, tableau 3, au lieu de “ intensité de l'effet en centimètres » lire ( intensité de l'effet en millimètres ). 